\title{
Analytic discrete cosine harmonic wavelet transform(ADCHWT) and its application to signal/image denoising
}

\author{
M. Shivamurti and S. V. Narasimhan \\ Digital signal processing and Systems Group \\ Aerospace Electronic and Systems Division, \\ National Aerospace Laboratories (CSIR), Bangalore-560017, INDIA \\ E-mail : narasim@nal.res.in
}

\begin{abstract}
A new simple and efficient analytic wavelet transform based on Discrete Cosine Harmonic Wavelet Transform DCHWT (ADCHWT) has been proposed and is applied for signal and image denoising. The analytic DCHWT has been realized by applying DCHWT to the original signal and its Hilbert transform. The shift invariance and the envelope extraction properties of the ADCHWT have been found to be very effective in denoising compared to that of DCHWT.

Key words: Analytic discrete cosine harmonic wavelet transform, Analytic wavelet transform, Dual tree complex wavelet transform, DCT, Shift invariant wavelet transform, Wavelet transform denoising
\end{abstract}

\section{Introduction}

The wavelet transform (WT) provides signal compression, denoising and many more desirable processing features. However, the WT coefficients oscillate about the zero value around the singularities.. This will reduce the magnitude of WT coefficients near singularity where they their are expected to be large, making the singularity extraction and signal modeling difficult. Further, the WT is shift variant, i.e., around singularities, even for a small shift in input signal, there will be a large variation in the energy distribution between WT coefficients at different scales resulting in different WT patterns which have to be considered for further processing [1]. This is due to aliasing caused by decimation at each wavelet level. Such a shift variant nature of WT not only affects detection of transients but also denoising as signal is reconstructed by decimated modified samples resulting in strong glitches [2]. Also at low signal to noise ratio like below $0 \mathrm{~dB}$, the conventional denoising fails. However the signal compression achieved by WT is not affected by its shift variant property.

The WT is realized by a perfect reconstruction filter bank which. involves analysis filter bank, down sampling, interpolation and synthesis filterbank. Here the aliasing caused by the use of nonideal filters is cancelled by the synthesis filter bank. However the reconstructed signal by such a filter bank is highly sensitive to coefficient errors and may get affected severely. Further such a WT suffers from poor directional selectivity for diagonal features. The WT filters being real, separable and their frequency response symmetric about zero in four quadrants in the $2 \mathrm{D}$ frequency space, cannot distinguish between two opposing diagonal directions, i.e. $+45^{\circ}$ and $-45^{\circ}$ edge orientations $[3,4]$.

The undecimated WT solves the shift variance with additional computational load. However the directionality problem remains as undecimated WT is blind to the two opposing diagonals as it uses separable filters. This blindness to directionality makes the processing and modeling of image features like ridges and edges difficult. For the separable filters to have proper directionality, their filter frequency responses should be asymmetric for positive and negative frequencies and can be achieved by using complex wavelet filters [4]. However the difficulty involved in the design of complex filters satisfying perfect reconstruction prohibits their use.

The sinusoids in the Fourier transform (FT) in higher dimensions provide highly directional plane waves. The FT magnitude: that is a smooth envelope extraction without oscillations, is shift invariant, has no aliasing problem and the signal reconstruction (inverse FT) does not involve any critical reconstruction criterion. These are due to its complex exponential basis instead of real basis of WT. Thus realization of complex/ analytic WT (AWT) has become important. This has the features of both WT and FT and is appropriate for time-frequency analysis like STFT [5]. This has been used for image segmentation, classification, deconvolution, image sharpening, motion estimation, coding, water marking [4,6], texture analysis and synthesis, seismic imaging and extraction of evoked potentials in EEG [7].

The AWT been realized using dual tree filter bank structure and Hilbert transform (HT). Variations of this structure have been achieved with various degrees of advantages and limitations. The absence of negative frequencies for an analytic signal not only reduces aliasing but also accounts for the decimation by a factor of 2 at each DWT stage resulting in shift invariance [3]. But the global nature of HT(infinite both time and frequency extent) converts the finite support wavelet to that of an infinite support, makes the shift invariance and alias free nature as approximate[3]. The WT realized by a two channel perfect reconstruction filter bank is computationally expensive and complicated due to explicit decimation, interpolation, associated filtering and involved delay compensation in reconstruction.

The harmonic wavelet transform (HWT) proposed by Newland [8] is simple and does not require 
explicit decimation, interpolation and associated filtering. The decimated components are achieved in the frequency domain by taking the inverse transform of each group of FT coefficients. The signal reconstruction is achieved by the inverse FT of properly concatenated FT coefficient groups. Here also, the WT coefficients become complex due to lack of DFT real symmetry. Further, the DFT values will be generally affected by the leakage due to signal truncation resulting in poor quality signal decomposition. To overcome these, the DCT based harmonic wavelet transform (DCHWT) has been proposed $[9,10]$. The real nature and the symmetrical signal extension of DCT respectively result in a real and more exact WT coefficients as the leakage is very much reduced [8]. The DCTHWT has been used for signal/ image compression and spectral estimation both with computational and performance advantage [9]. With this, efficient and accurate signal decomposition for crossterm suppression in Wigner-Ville time frequency distribution [10] and reduced glitches denoising is achieved by extended it to shift invariant version.[2]. It is important to note that the complex arithmetic computational load is justified by its many advantages.

In the present work, a new analytic wavelet transform based on DCHWT (ADCHWT) has been proposed and is applied for signal and image denoising. The analytic DCHWT has been realized by applying DCHWT to the original signal and its HT. The shift invariance property of the ADCHWT has been illustrated and its contribution in association with its envelope extraction property has been found to be very effective in denoising compared to that of DCHWT.

\section{Discrete Cosine Harmonic Wavelet Transform (DCHWT)}

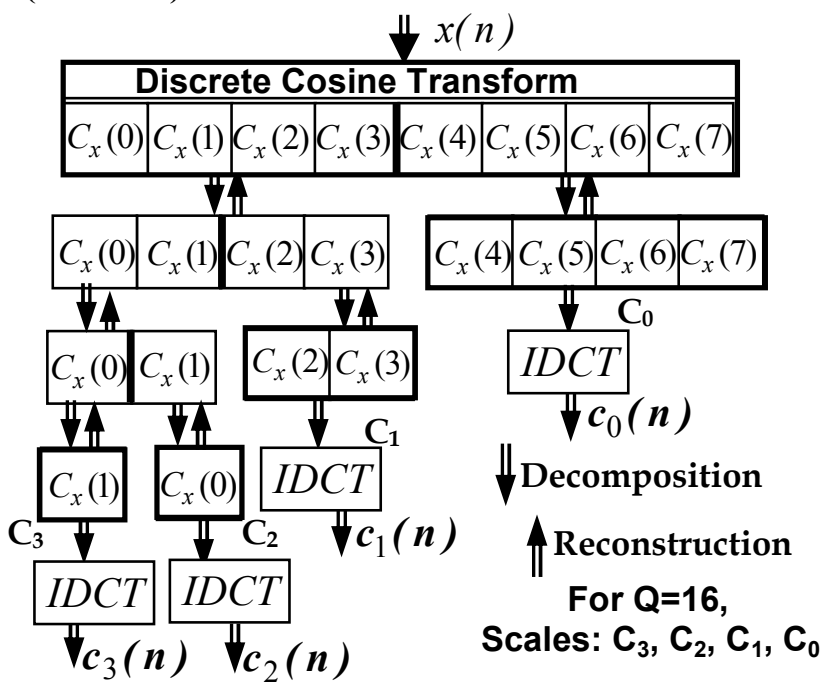

\section{Fig.1. DCHWT for a 1-D signal}

The filter bank realization of WT, involves down sampling of the components obtained by the individual bandpass filters. The restoration of the processed signal corresponding to overall spectrum at the original sampling rate, involves summation of the components after their upsampling and image rejection filtering. The harmonic wavelet transform based on DFT (DFHWT) realizes the subband decomposition in the frequency domain by grouping the Fourier transform (FT) coefficients and the inverse of these groups results in decimated signals [7]. Further after processing, the FT of the subband signals can be repositioned in their corresponding positions to recover the overall spectrum, with the original sampling rate. Therefore, this will not involve explicit decimation and interpolation operations. As a consequence, no band limiting and image rejection filters are necessary. Also, while reconstruction, there are no delay compensations as the subband groups are synthesized in frequency domain by repositioning them. In view of this, the harmonic wavelet decomposition is very attractive due to its simplicity. However in the DFHWT, the grouping of the DFT coefficients with lack of conjugate symmetry makes the WT coefficients complex. For reconstruction after concatenation of the groups, the conjugate symmetry is restored to get the real signal.

The DFHWT is very attractive as long as no processing of the components is involved prior to inverse transformation. If different subbands have to be processed differently, this is not achieved as the signal energy from one to another has already leaked. The DFHWT may be tolerable for a signal with wellseparated frequency components of sufficiently high magnitude. But for closely spaced components of significantly different magnitudes, during the computation of the FT itself with out using a smoothing window, the energy will leak from the higher amplitude component to the lower one (and vice versa) resulting in a large bias in the spectral magnitude which may even totally eclipse smaller amplitude spectral peaks. This will also limit its use for compression as not possible to get a good signal reconstruction by omitting the lower scales (corresponding to high frequencies) in WT as the leaked energy cannot be recovered unless all the scales are considered. Therefore to utilize the advantages of the harmonic WT, DCT which has a comparatively reduced leakage effect is used instead of DFT.. This is due to symmetrical extension of data in DCT which results in a smooth transition from one period to the other without any discontinuity [8].

The DCT coefficients are grouped in a way similar to that of DFT coefficients and the DCT being real, the conjugate operation is not necessary.in placing the coefficients symmetrically[9]. The grouped coefficients for each band have to be treated as if they are the DCT coefficients of that subband.

In Fig.1 DCTHWT for a DCT size of $Q=16$ is illustrated and the only one side of the symmetrical coefficient sequence is shown, i.e. (0-7). The last half part of the coefficients $C_{x}(4)$ to $C_{x}(7)$ correspond to scale- $0, C_{0}$. The lower half of the coefficients $C_{x}(0)$ to $C_{x}(3)$ are split into two groups and the upper group $\left[C_{x}(2), C_{x}(3)\right]$ correspond to scale- $1, C_{1}$. Further 
the group $\left[C_{x}(0), C_{x}(1)\right]$ is split into two groups $C_{2}$ and $C_{3}$ each having single coefficients $C_{x}(1)$ and $C_{x}(0)$, respectively. The inverse DCT of each of the groups $C_{0}, C_{1}, C_{2}, C_{3}$; result in WT coefficients of the scales $0,1,2,3$, respectively.

For the reconstruction, $C_{3}, C_{2}, C_{1}, C_{0}$, the DCTs of the subband signals are concatenated to get the DCT of the fullband signal (Fig.3(a)). and its inverse DCT provides the reconstructed signal.

\section{Analytic Discrete Cosine Harmonic wavelet transform (ADCHWT)}

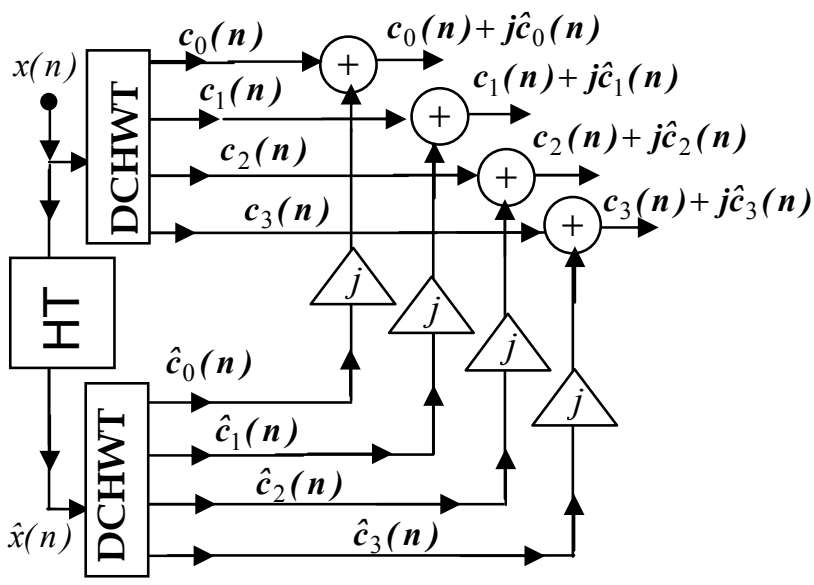

\section{Fig.2. Schematic of ADCHWT for a 1D signal}

(a) 1D signal : There are different methods of obtaining the AWT which uses the DWT. The straight forward post filtering approach splits each filterbank output into positive and negative frequency components using a complex PRFB acting as a HT though looks simple suffers from nonzero values in the negative frequency region [1]. The dual tree complex wavelet transform uses two DWT trees one for the real part of the analytic signal and the other for its imaginary part, the HT of the input. The corresponding scales of the two trees are combined to achieve the desired analytic WT. This does not require any complex filters and suffer from any performance limitation but its computational load is twice that of a DWT as two DWT trees. The proposed ACHWT is also realized by a dual tree approach and is shown in the Fig. 2 for 4 scales. Here the DCHWT 4 scales for the original signal are obtained in the method explained in section 2.1. The input signal is converted to its HT and again its DCHWT 4 scales are obtained. Thus the different scales and their HTs are obtained by simply converting the input to its HT. Further, Hilbert transformed scales are weighted by $j$ and are combined with their corresponding scales by summation to get the analytic harmonic discrete cosine wavelet transform (ADCHWT). For reconstruction, the real part of the ADCHWT is taken and the procedure is same as given in Section.2.1.

The HT forms an integral part of the ADCHWT and hence its quality determines the performance of ADCHWT. The analytic signal can be realized in the frequency domain by making the negative frequency components of the original signal to zero and scaling the positive components by a factor 2and taking its inverse FT. The imaginary part of this analytic signal gives the desired HT of the signal. However, this method suffers from leakage problem of DFT as energy from negative frequency components would have leaked into positive frequency region. Hence, it is desirable to realize HT of the signal in time domain by convolving the HT impulse response with the input signal [8]. Impulse response of HT, $h(n)=\left\{2 \sin ^{2}(\pi n / 2)\right\} / n \pi,-\infty \leq n \leq \infty$. To avoid Gibbs effect in truncating of the HT impulse response, it is windowed by a smoothing window. The shift invariance performance of ADCHWT is illustrated for an impulse and a rectangular pulse. The magnitude difference of the WT coefficients (for different scales) between original signal and its shifted version with shift of 4 samples are plotted (Fig. 3 shown in last page of the paper) For ADCHWT, this difference is small compared to that of DCHWT as the latter gets affected by the shift. The difference energy indicated on the top of each subgroup, Fig. 3 also supports the shift invariance property of ADCHWT as it is relatively small compared to that of DCHWT

(b) 2D signal : For a 2D signal [10], the DCT coefficients for the columns are split in to two groups and their inverse DCT results in DCTHWT coefficients for the columns. The DCT along the rows for each scale are taken and grouped. The inverse DCT of these groups will result in 2D DCTHWT (Fig.4). This procedure is repeated for further scales considering the LL block as input. The procedure holds good for the real part of ADCHWT of an image.

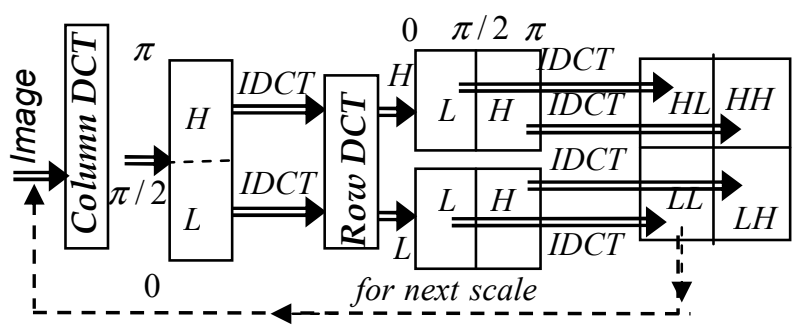

Fig.4. ADCT for a 2D signal ( real part)

For the imaginary part of ADCHWT image on hand, its HT has to be considered. For this to start with, prior to taking the DCT, the HT has to be taken. That is, the HT along the columns of the image are taken and for these Hilbert transformed columns, the DCTs are taken. Further the DCT coefficients are grouped in to two groups and for each group, inverse DCT is applied to get the WT scales corresponding to the image columns. For each scale along the rows, the HTs are taken and for Hilbert transformed rows, the DCTs are taken. Again these DCT coefficients are grouped in to two groups and for each group inverse DCT is applied to get ADCHWT with scales HL, HH, LL and LH . As the HT has to be applied column and row wise only once, for higher scales, that is for splitting LL further, HT should not be applied and hence is similar to that of the real part decomposition. 


\section{ADCHWT Denoising}

In WT denoising, unlike removal of frequency components in FT based methods, no (higher) frequency component is removed which results in smoothing of fast changes or edges. Here denoising is done in time domain and hence no scale / frequency is removed unless it is totally not contributing to the signal. In WT denoising, in each scale, those values which are below a certain threshold are made zero/ modified and the signal is reconstructed with these modified scales. Here this noise is assumed to be distributed over all scales equally and their magnitudes will be small. But the problem with WT denoising is its shift variant nature. In WT domain as the signal is reconstructed with modified decimated scale samples, there may be glitches as in between samples are removed especially at higher scales [11]. The undecimated WT overcomes this but at the expense of additional computational load. Hence, the analytic WT which provides shift invariance due to its reduced bandwidth by a factor of half [3], is an appropriate solution. Here in denoising, the threshold for each scale is found by using the absolute values of ADCHWT. Further, the absolute values of ADCWT are compared with the threshold to decide whether a particular WT coefficient has to be retained / modified and is applied to DCHWT. Here not only the ADCWT's shift invariant nature but also its good envelope extraction contributes. This DCHWT is used for the signal reconstruction. Hence sometimes, ADCWT denoising performs better than those of shift invariant WT.

\section{Simulation Results and Conclusions}

The performance of the ADCHWT is shown for a discontinuous signal ( $\mathrm{SNR}=9 \mathrm{~dB})$, speech segment $(\mathrm{SNR}=10 \mathrm{~dB})$ and an image $(\mathrm{SNR}=22 \mathrm{~dB})$. The noise associated with these is zero mean white Gaussian noise. For the general signal, 11 scales and for speech, 10 scales are considered. In both cases denoising is done for lower 5 scales. For image, 3 levels of decomposition are considered and denoising is done for all scales in all levels except for level-3 scale-1 (LL). In all these cases, hard thresholding is applied using scaled standard deviation of the first scale as the threshold. The standard deviation scaled by a factor 6 and 7 for the general signal, and speech, respectively. For image, a threshold value of 70 which corresponds to minimum error energy between the original and reconstructed image, is found experimentally. For the discontinuous signal, the sharpness at discontinuous points is maintained better compared to that of DCHWT and is reflected in their respective output SNRs (13.1 and $13.7 \mathrm{~dB}$ (Fig.5(c, d)). For the speech, ADCHWT extracts the signal envelope well resulting in a better output SNR by $4 \mathrm{~dB}$ compared to that of DCHWT (Fig.6(c, d)) however both sound some what equal for hearing. For the image, again for the ADCHWT, the output SNR is better by $1.2 \mathrm{~dB}$ compared to that of DCHWT (Fig.7(c, d)).

A new Analytic Cosine Harmonic Wavelet transform was proposed. Its shift invariant property with respect to its real counter part was found to be superior both for impulse and pulse signals. Its application to noisy discontinuous signal, speech and image indicated that due to its shift invariant and envelope preserving properties in deciding the modification of WT has resulted in a superior performance than its real version. The real nature and the built in decimation and interpolation without any explicit filtering and delay compensation, makes the new algorithm simple and computationally efficient compared to other dual tree analytic algorithms.

\section{References}

[1] I.W. Selesnick, R.G. Baraniuk and N.G.Kinsbury, The dual tree complex wavelet transform., IEEE Signal Processing Magazine, November 2005.pp123-151

[2] S.V.Narasimhan and Aniruddha Adiga "Shift Invariant Discrete Cosine Harmonic Wavelet Transform and Its Application to Denoising, IEEE INDICON 2007.Bangalore.

[3] E. Hostalkova and A. Prochazka, "Complex wavelet transform in biomedical image denoising" In Proceedings of 15th Annual Conference Technical Computing Prague 2007, pages $\mathrm{HP} / 1-8$,

[4 Natasa Terzija and Walter Geisselhardt, Digital water image marking using complex wavelet transform, International Multimedia Conference, Proceedings of the workshop on Multimedia and security, 2004, pp.193198,Magdeburg, Germany

[5] X. Zhu and J. Kim, Application of analytic wavelet transform to analysis of highly impulsive noises, Journal of sound and vibration, 294,2006, pp8941-855.

[6] L.Hongxing, C Songqiao, L/Taoshen, H.Aina A digital water marking algorithm based on dual tree complex wavelet transform, IEEE $9^{\text {th }}$ International conference for Young Computer Scientists, 2008, pp.1488-1492

[7] A.Jacquin, E.Causevic, Roy John, Jelena Kovacevic, Adaptive comples wavelet based filtering of EEG for extraction of evoked potential responses., Inter. Conf. on Acoustics, Speech and Signal Processing, 2005, pp V393-396.

[8] D.E. Newland, "Harmonic Wavelet analysis", Procee. of Royal Society, London-A, Vol. 444, 1993, pp. 203-225.

[9] S.V. Narasimhan and M. Harish, "A new spectral estimator based on discrete cosine transform and modified group delay", Signal Processing, Vol.86, 2006, pp.1586-1596.

[10] S. V. Narasimhan, M. Harish, A. R. Haripriya, Nandini Basumallick, "Discrete cosine harmonic wavelet transform and its application to signal compression and subband spectral estimation using modified group delay", Signal, Image and Video Processing, Vol. 3., No.1, Feb. 2009, pp. 85-99.

[11] S.V. Narasimhan, A.R.Haripriya, B. K. Shreyamsha Kumar, "Improved wigner-ville distribution performance based on DCT/DFTharmonic wavelet transform and modified magnitude group delay", Signal Processing, Vol.88, 2008, pp. 1-18. 

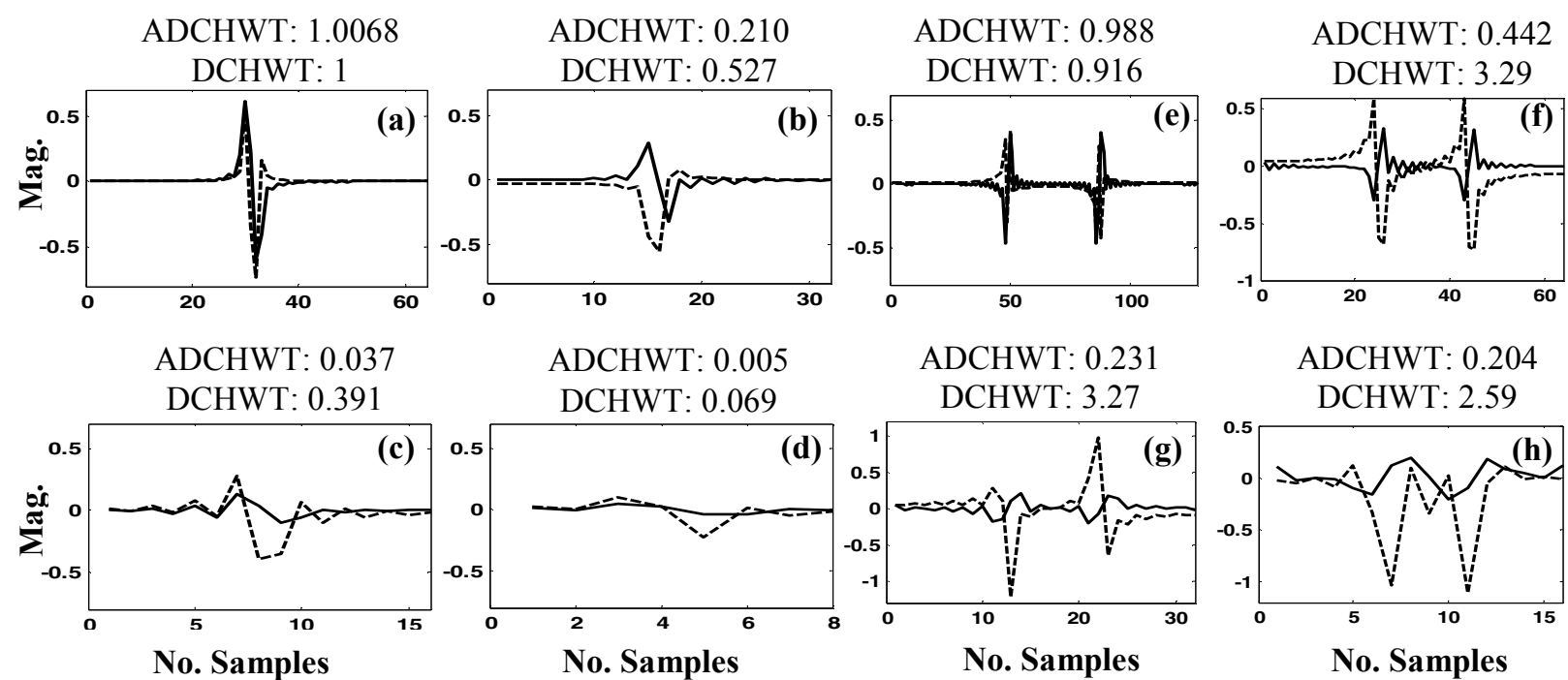

Fig.3. Difference in WT coeff along with energy for Impulse and pulse on top. - - - DCHWT, - ADCHWT For impulse :(a) Scale0,(b)Scale1 (c)Scale2 (d) Scale3 and for pulse : (e) Scale0,(f)Scale1 (g)Scale2 (h) Scale3

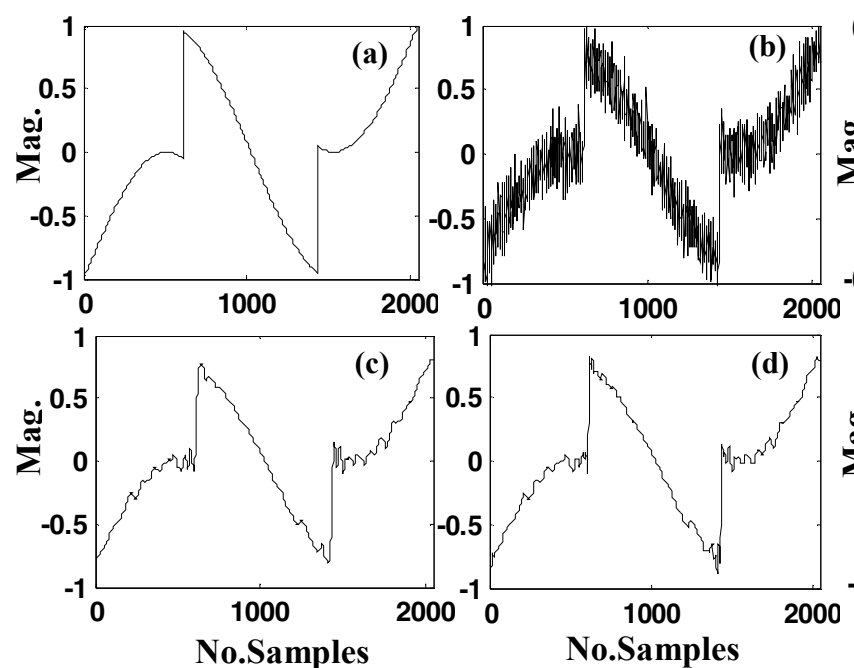

Fig.5 Denoising comparison for discontinuous signal (a) Clean (b) Noisy (10dB) (c) by DCHWT,O/P$\mathrm{SNR}=13.1 \mathrm{~dB}$ (d) ADCHWT,O/P-SNR $=13.7 \mathrm{~dB}$
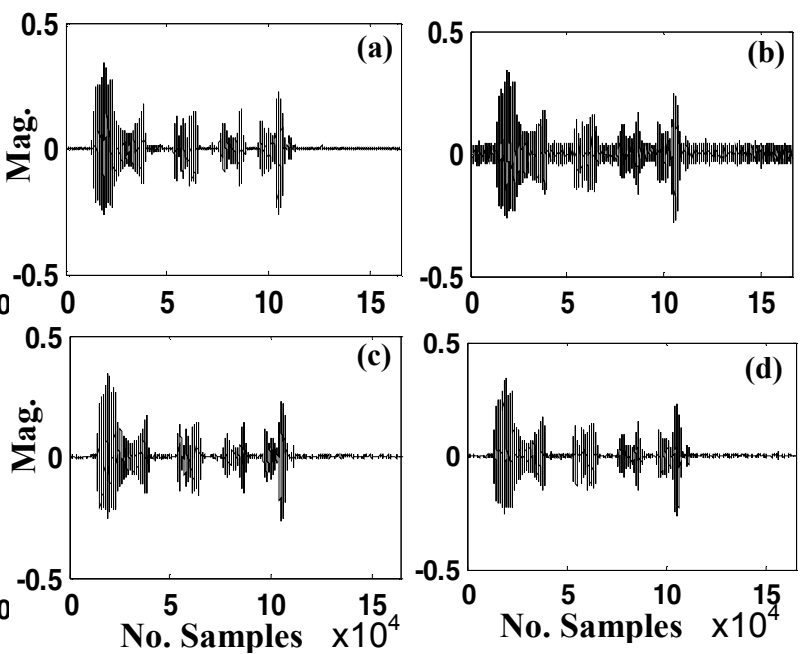

(c)

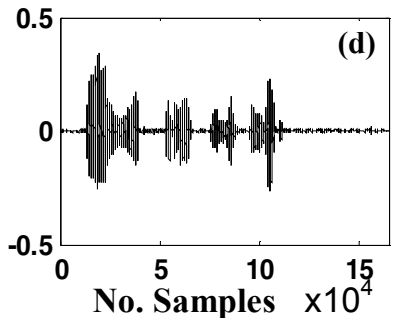

Fig. 6 Denoising Comparison for speech

(a) Clean (b) Noisy (9dB) (c) DCHWT, O/PSNR $=14 d B$ (d) ADCHWT, O/P-SNR $=18 d B$

(a)

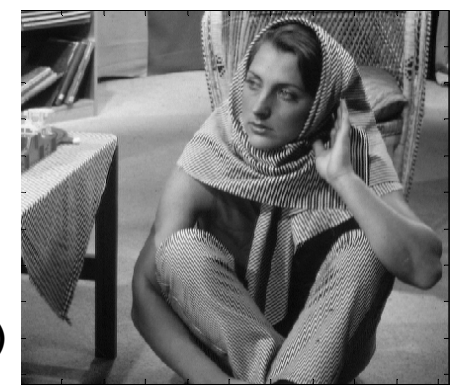

(c)

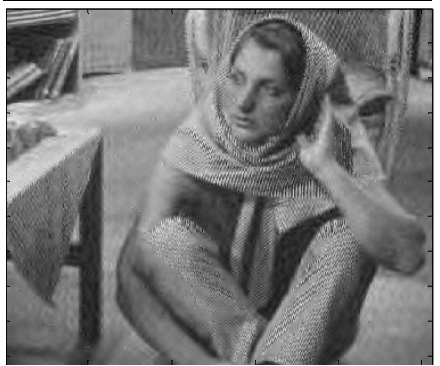

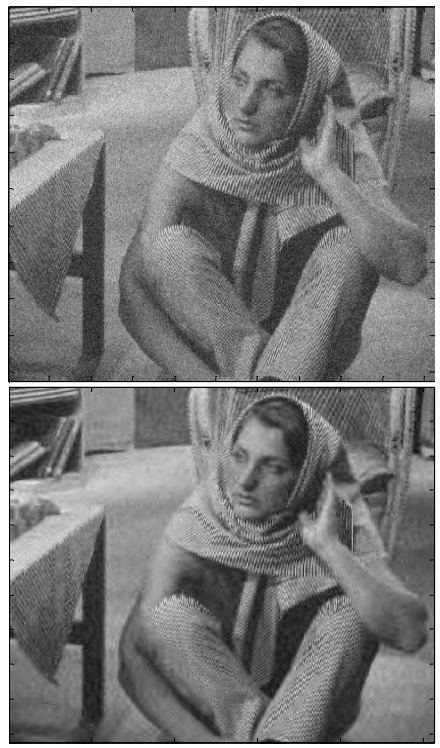

Fig.7 Comparison of DCHWT and ADCHWT for image with I/P-

(b) $\quad \mathrm{SNR}=22 \mathrm{~dB}$.
(a) Clean Image (b) Noisy image
(c) DCHWT,O/P-SNR=18.3dB
(d) $\mathrm{ADCHWT}, \mathrm{O} / \mathrm{P}-\mathrm{SNR}=19.5 \mathrm{~dB}$

(d) 